\title{
Combinatorial Functions of Transcription Factors and Epigenetic Factors in Heart Development and Disease
}

\author{
Kazuko Koshiba-Takeuchi, Yuika Morita, Ryo Nakamura, \\ and Jun K. Takeuchi
}

\begin{abstract}
Heart malformations are the most common type of birth defect, affecting more than $2 \%$ of newborns and causing significant morbidity and mortality. In the past two decades, studies have revealed the function and importance of cardiac transcription factors during heart development and in congenital heart disease. Transcription factors generally form complexes with other transcription factors and/or with chromatin factors to perform specific functions. This review focuses on how chromatin factors modify cardiac transcription factors during cardiovascular development and disease.
\end{abstract}

\section{Keywords}

Cardiac development - Cardiac disease • T-box genes • Epigenetic factors • $\mathrm{SWI} / \mathrm{SNF}$-type chromatin remodeling factors

K. Koshiba-Takeuchi

Department of Integrated Biosciences, Graduate School of Frontier Science, Tokyo, Japan

Department of Biological Sciences, Graduate School of Sciences, The University of Tokyo, Tokyo, Japan

Y. Morita • R. Nakamura

Department of Integrated Biosciences, Graduate School of Frontier Science, Tokyo, Japan

JST PRESTO, Tokyo, Japan

J.K. Takeuchi $(\square)$

Department of Integrated Biosciences, Graduate School of Frontier Science, Tokyo, Japan

Department of Biological Sciences, Graduate School of Sciences, The University of Tokyo, Tokyo, Japan

JST PRESTO, Tokyo, Japan

e-mail: junktakeuchi@iam.u-tokyp.ac.jp

T. Nakanishi et al. (eds.), Etiology and Morphogenesis of Congenital Heart Disease,

DOI 10.1007/978-4-431-54628-3_42 


\subsection{Transcription Factors in Heart Development}

The heart is an organ that pumps blood to and from the body's tissues through the blood vessels. Cardiac muscle contains cardiomyocytes, which ensure the heart's contractile ability; however, cardiomyocytes alone are not sufficient for the heart to function. Other components, such as the conduction cells, fibroblasts, blood vessels, and endocardial cells, are important for maintaining the heart's systemic pumping ability. Each cell type can be identified by its expression of specific transcription factors, signaling molecules, and/or function-specific proteins (Fig. 42.1). The heart is the first organ to form in vertebrates, and it performs a vital role in distributing oxygen and nutrients throughout the embryo. The primordial heart is derived from cardiovascular mesodermal cells that transiently express T (Brachyury), Mespl, and Flkl during gastrulation. A subset of these cardiac mesodermal cells gives rise to cardiac progenitor cells, which can differentiate into any type of cardiac cell.

T-box transcription factors compose a conserved family of genes that are important for heart development and patterning. In humans, disruption of the cardiac T-box genes leads to various congenital heart defects [1]. Mutations in $T b x 5$ are associated with Holt-Oram syndrome [2, 3], whereas mutations in the $T b \times 20$ gene result in atrial septal defect $[4,5]$. Interestingly, in the developing heart, Tbx5 and Tbx20 are complementarily expressed in the left and right ventricle, respectively (Fig. 42.2) [6]. The regions in which the $T$-box genes are expressed and the regions that are defective in a given disease are very similar. $T b x 5$ is expressed in the inflow tract, atria, AV cushion, and left ventricle but not in the outflow tract or

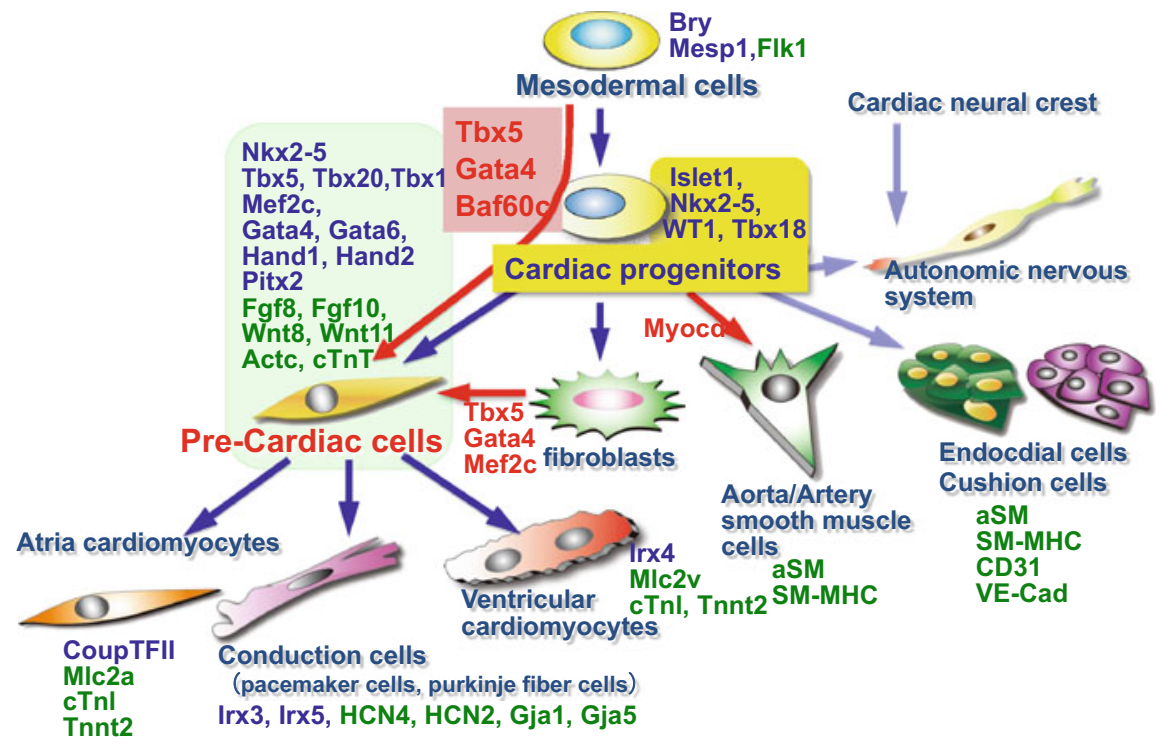

Fig. 42.1 Multiple cardiac cell types. Each cell type differentiates from mesoderm-derived cardiac progenitor cells. The major molecules associated with each cell type are indicated 


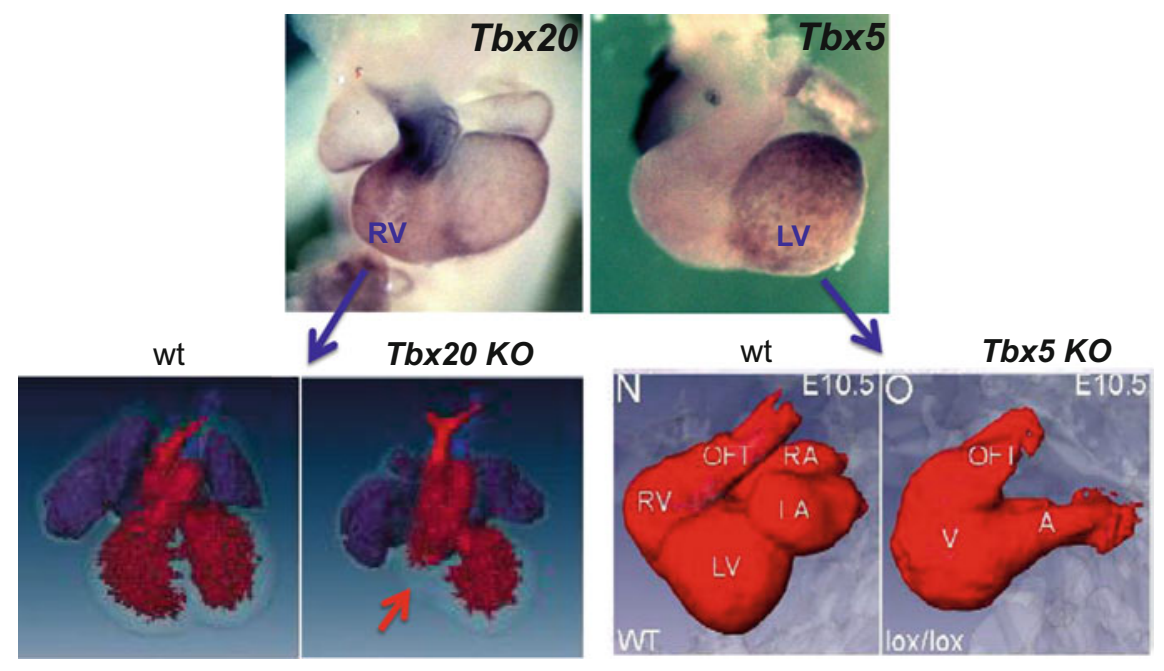

Fig. 42.2 The expression patterns of $T b x 5$ and $T b x 20$ in the mouse heart. These genes show complementary expression patterns in the ventricles. The knockout mice for each gene show hypoplasia in the same region in which the gene is normally expressed (Adapted from Bruneau et al. [8] and Takeuchi et al. [9])

the right ventricle; $T b x 5$ expression appears to be restricted to the first heart field (FHF)-derived region [7]. Tbx5 knockout mice experience severe left ventricle hypoplasia and die at approximately E9 without their hearts ever beating (Fig. 42.2) [8]. By contrast, Tbx20 is primarily expressed in the outflow tract and the right ventricle, which are derived from the second heart field (SHF). Tbx20 knockdown mice develop a single ventricle and show severe hypoplasia of the right ventricle (Fig. 42.2) [9]. These facts indicate that Tbx5 and Tbx20 may specify the identity of each ventricle. Tbx5 also acts in association with Sall4 in ventricular septum formation. Sall4 is a zinc-finger transcription factor that, when mutated, causes Okihiro syndrome (Duane-radial ray syndrome, DRRS) in humans $[10,11]$. The heart and limb phenotypes of Okihiro syndrome are very similar to those of Holt-Oram syndrome. In fact, some Holt-Oram patients lack mutations in TBX5 and instead have mutations in SALL4 [12]. Tbx5 and Sall4 participate in protein-protein interactions and synergistically regulate downstream gene expression [13]. Furthermore, Tbx5 is a key gene involved in the acquisition of the ventricular septum during vertebrate evolution [14]. During vertebrate evolution from aquatic to terrestrial life, the morphology of the heart has changed. As a result, avian and mammalian hearts contain four chambers-two atria and two ventricles - and their circulatory systems contain two loops, the pulmonary and systemic loops, that separate the oxygen-rich and oxygen-poor blood. In vertebrates with four-chambered hearts, Tbx5 expression is restricted to the left ventricle, whereas in animals with a single ventricle, $T b x 5$ expression is observed throughout the ventricle. Reptiles show a unique $T b x 5$ expression pattern that is associated with 


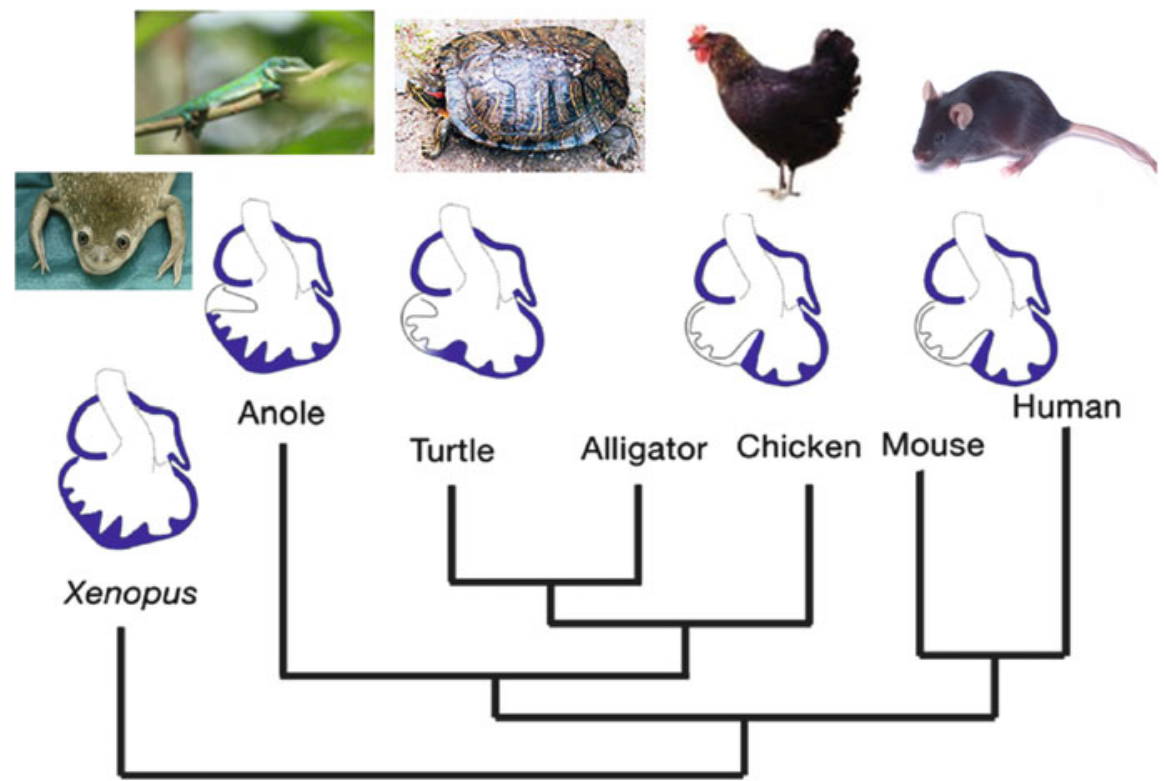

Fig. 42.3 $T b x 5$ expression and heart morphology in vertebrates. Note that animals with two ventricles express $T b \times 5$ on the left side (Adapted from Koshiba-Takeuchi et al. [14])

their ventricular morphology. Anolis, which are a type of squamate, have a single ventricle that expresses $T b x 5$ throughout this chamber and throughout development. Interestingly, turtles show a left-high to right-low gradient of $T b x 5$ expression during late developmental stages, and a septum-like structure forms in the middle of the ventricle (Fig. 42.3). To confirm the precise interaction between Tbx5 expression patterns and ventricular septum formation, we performed Tbx 5 mis-expression experiments using transgenic mice. Transgenic mice that express Tbx 5 throughout the ventricle fail to form a ventricular septum. These results strongly indicate that Tbx5 expression in the left ventricle is important for the development of two-chambered ventricles. We hypothesized that the regulatory region of $T b x 5$ might have been modified during vertebrate evolution, thereby changing the $T b x 5$ expression pattern and the ventricular morphology, as shown in Fig. 42.3.

\subsection{Chromatin Factors and Cardiac Differentiation}

Recent studies have shown that chromatin factors are essential for determining cell fate in several organs. In heart development, SWI/SNF-type chromatin remodeling factors play key roles in the differentiation of cardiomyocytes by interacting with heart-specific transcription factors $[15,16]$. Cardiac transcription factors alone are not sufficient to induce cardiomyocyte differentiation in vivo or in vitro (Figs. 42.4 and 42.5). This result suggests that chromatin accessibility is important for 

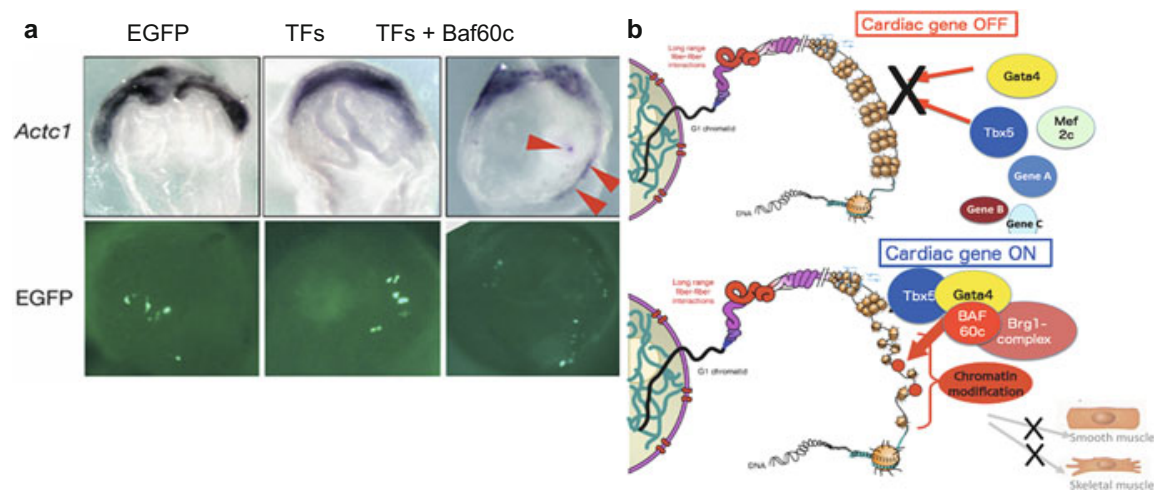

Fig. 42.4 (a) A mixture of transcription factors (TFs), Baf60c and EGFP, but not the control (EGFP) or TFs + EGFP, ectopically induce Actcl in the lateral plate mesodermal region. (b) A schematic of cardiac gene regulation. The SWI/SNF complex-mediated change in chromatin conformation is important for the activation of cardiac gene transcription (Adapted from Takeuchi and Bruneau [18] and Van Weerd et al. [15])

\begin{tabular}{cccccccc}
\hline Tbx5 & Gata4 & Gata1 & Nkx2-5 & Baf60c & Baf60b & $\begin{array}{c}\text { Actc1,Myl7 } \\
\text { expression }\end{array}$ & Beating \\
+ & - & - & - & - & - & $\times$ & $\times$ \\
\hline- & + & - & - & - & - & $\times$ & $\times$ \\
\hline- & - & + & - & - & - & $\times$ & $\times$ \\
\hline+ & + & - & - & - & - & $\times$ & $\times$ \\
\hline+ & - & + & - & - & - & $\times$ & $\times$ \\
\hline- & - & - & + & - & - & $\times$ & $\times$ \\
\hline+ & - & - & + & - & - & $\times$ & $\times$ \\
\hline- & + & - & + & - & - & $\times$ & $\times$ \\
\hline+ & + & - & + & - & - & $\times$ & $\times$ \\
\hline- & + & - & - & + & - & 0 & $\times$ \\
\hline- & + & - & - & - & + & 0 & $\times$ \\
\hline- & - & + & - & + & - & 0 & $\times$ \\
\hline- & - & + & - & - & + & $\times$ & $\times$ \\
\hline+ & + & - & + & + & - & 0 & $\bigcirc$ \\
\hline+ & + & - & - & + & - & $\bigcirc$ & $\bigcirc$ \\
\hline
\end{tabular}

Fig. 42.5 Schematic diagram shows that ectopic expression of major cardiac contracted genes (Actc1 and Myl7) are observed by combinatorial transfection of cTF and Baf60c into ex vivo mouse 


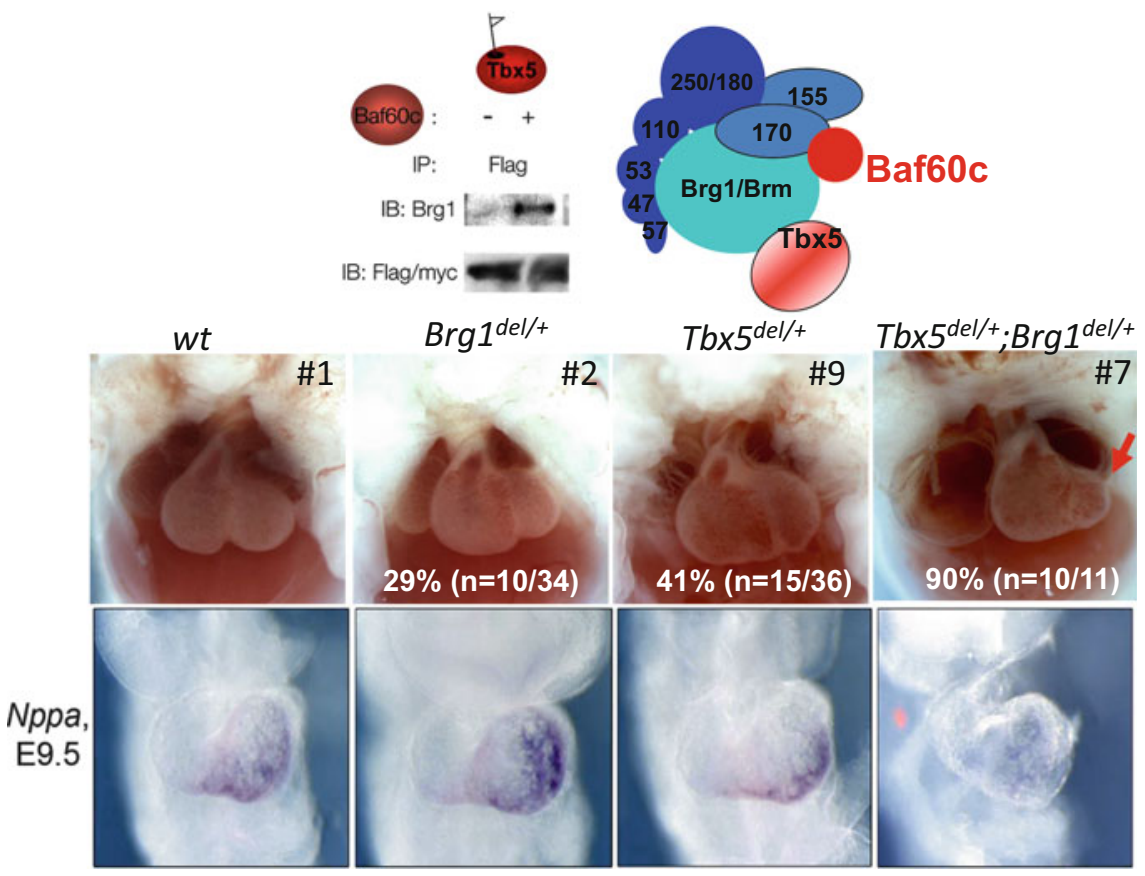

Fig. 42.6 The combinatorial functions of cardiac transcription factors and Baf chromatin remodeling factors. Only the transcription factors can induce cardiac markers, but the differentiation of beating cardiomyocytes requires both transcription factors and chromatin remodelers

transcription factors to bind to their target sites. Therefore, we searched for chromatin remodeling factors that are expressed in the cardiac region at early stages of heart development. A previous study showed that a component of the SWI/SNFtype chromatin remodeling complex, Baf60c (also known as Smarcd3), has specific roles in heart development [17]. When a mixture of cardiac transcription factors and Baf60c was injected into the lateral plate mesoderm of mouse embryos, alpha cardiac actin-positive cells were ectopically induced (Fig. 42.4) [18]. These ectopically induced cardiac cells could beat, which showed that they were functional cardiomyocytes. Baf60c can directly associate with the Tbx5, Nkx2-5, Gata4, and RBPjk proteins and regulate the transcription of downstream genes $[17,19]$. Mutations in chromatin factors cause abnormal cardiac function in both mice and humans. Baf60c determines a cell's fate by not only loosening the chromatin structure but also synergistically interacting with specific factors. When associated with Tbx5, Brg1, a core protein of the SWI/SNF-type chromatin remodeling complex, synergistically regulates cardiac differentiation in the presence of Baf60c [20]. Mice heterozygous for both Brgl and Tbx5 had more severe defects than did single mutants, particularly in the left ventricle (Fig. 42.6). These 
results indicate that the dosage of epigenetic factors affects the severity of the Tbx5 mutant phenotype (i.e., left ventricular hypoplasia). The severity of congenital heart disease in humans may be related to the level of expression of epigenetic factors and/or of partner factors. To address this possibility, we need to elucidate the relationship between the expression level of epigenetic factors and the penetration of heart failure.

\subsection{Future Directions and Clinical Implications}

We have analyzed the functions of Baf60c, a component of the SWI/SNF-type chromatin remodeling complex, in heart development in vivo and in vitro, but the mechanism by which Baf60c is regulated is still unknown. An important question is whether the functions of $\mathrm{Brg} 1$ or Baf60c are altered in each type of tissue. If their functions do not vary, they may be regulated in a partner-dependent manner. One approach to confirm this hypothesis is to use ChIP-sequencing to compare Baf60c and $\mathrm{Brg} 1$ target genes in different tissues. Another question that must be addressed is how Baf60c's expression pattern and dosage are regulated and which molecule (s) participate in this regulation. We found a candidate transcription factor that directly regulates Baf60c expression, but this molecule alone could not explain the dynamic change in Baf60c's expression pattern. Further analysis is required to determine the molecular mechanisms of Baf60c regulation.

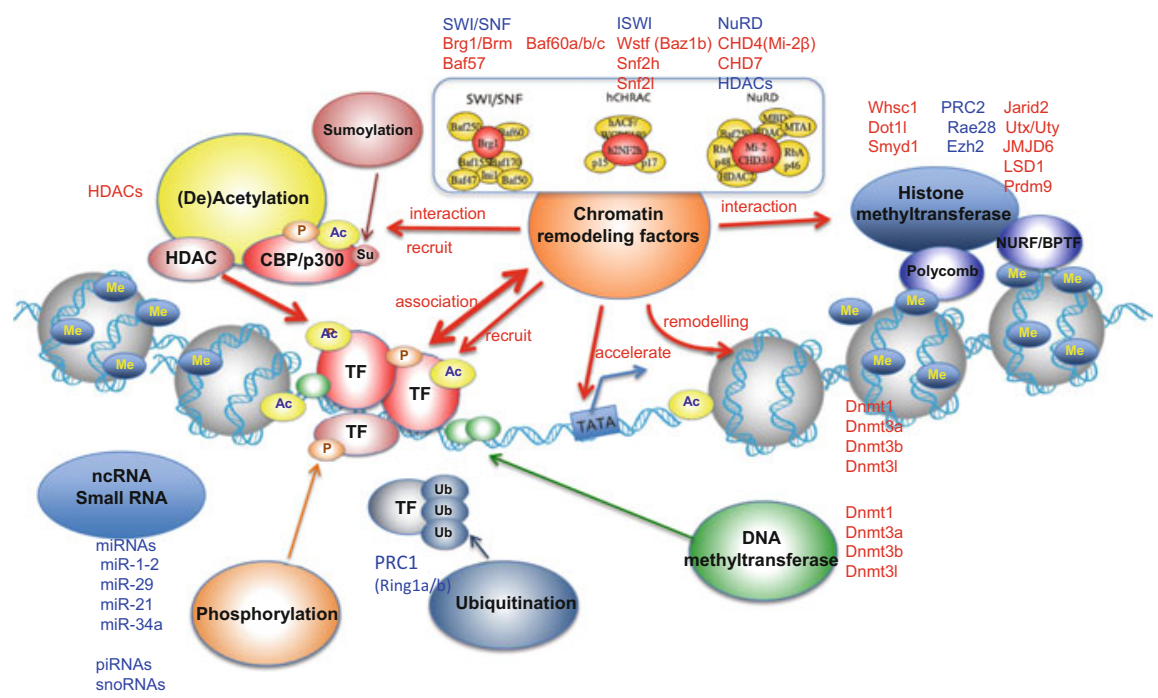

Fig. 42.7 Immunoprecipitation experiments indicate that $\mathrm{Brg} 1$ can strongly bind to $\mathrm{Tbx} 5$ with Baf60c. The lower panel shows the morphology of Brgldel/+, Tbx5del/+, and double heterozygote mouse hearts. The heart of the double heterozygote shows severe hypoplasia of the left ventricle (Adapted from Takeuchi et al. [20]) 
Over the last 20 years, cardiac researchers have elucidated many causes of congenital heart disease and have identified many genes that are involved. However, it is not sufficient to only understand the diversity or severity of the disease. In the future, we must also determine the role of epigenetic factors in heart failure because these factors regulate cardiac gene transcription (Fig. 42.7).

Open Access This chapter is distributed under the terms of the Creative Commons AttributionNoncommercial 2.5 License (http://creativecommons.org/licenses/by-nc/2.5/) which permits any noncommercial use, distribution, and reproduction in any medium, provided the original author(s) and source are credited.

The images or other third party material in this chapter are included in the work's Creative Commons license, unless indicated otherwise in the credit line; if such material is not included in the work's Creative Commons license and the respective action is not permitted by statutory regulation, users will need to obtain permission from the license holder to duplicate, adapt or reproduce the material.

\section{References}

1. Greulich F, Rudat C, Kispert A. Mechanisms of T- box gene function in the developing heart. Cardiovasc Res. 2011;91:212-22.

2. Li QY, Newbury-Ecob RA, Terrett JA, et al. Holt-Oram syndrome is caused by mutations in TBX5, a member of the Brachyury (T) gene family. Nat Genet. 1997;15:21-9.

3. Basson CT, Bachinsky DR, Lin RC, et al. Mutations in human TBX5 cause limb and cardiac malformation in Holt-Oram syndrome. Nat Genet. 1997;15:30-5.

4. Kirk EP, Sunde M, Costa MW, et al. Mutations in cardiac T-box factor gene TBX20 are associated with diverse cardiac pathologies, including defects of septation and valvulogenesis and cardiomyopathy. Am J Hum Genet. 2007;81:280-91.

5. Posch MG, Gramlich M, Sunde M, et al. A gain-of-function TBX20 mutation causes congenital atrial septal defects, patent foramen ovale and cardiac valve defects. J Med Genet. 2010;47:230-5.

6. Takeuchi JK, Ohgi M, Koshiba-Takeuchi K, et al. Tbx5 specifies the left/right ventricles and ventricular septum position during cardiogenesis. Development. 2003;130:5953-64.

7. Bruneau BG, Logan M, Davis N, et al. Chamber-specific cardiac expression of Tbx5 and heart defects in Holt-Oram syndrome. Dev Biol. 1999;211:100-8.

8. Bruneau BG, Nemer G, Schmitt JP, et al. A murine model of Holt-Oram syndrome defines roles of the $\mathrm{T}-$ box transcription factor $\mathrm{Tbx} 5$ in cardiogenesis and disease. Cell. 2001;106:709-21.

9. Takeuchi JK, Mileikovskaia M, Koshiba-Takeuchi K, et al. Tbx20 dose-dependently regulates transcription factor networks required for mouse heart and motoneuron development. Development. 2005; 132:2463-74.

10. Al-Baradie R, Yamada K, St Hilaire C, et al. Duane radial ray syndrome (Okihiro syndrome) maps to 20q13 and results from mutations in SALL4, a new member of the SAL family. Am J Hum Genet. 2002;71:1195-9.

11. Kohlhase J, Heinrich M, Schubert L, et al. Okihiro syndrome is caused by SALL4 mutations. Hum Mol Genet. 2002;11:2979-87.

12. Brassington A-ME, Sung SS, Toydemir RM, et al. Expressivity of Holt-Oram syndrome is not predicted by TBX5 genotype. Am J Hum Genet. 2003;73:74-85.

13. Koshiba-Takeuchi K, Takeuchi JK, Arruda EP, et al. Cooperative and antagonistic interactions between Sall4 and Tbx5 pattern the mouse limb and heart. Nat Genet. 2006;38:175-83. 
14. Koshiba-Takeuchi K, Mori AD, Kaynak BL, et al. Reptilian heart development and the molecular basis of cardiac chamber evolution. Nature. 2009;461:95-8.

15. van Weerd JH, Koshiba-Takeuchi K, Kwon C, Takeuchi JK. Epigenetic factors and cardiac development. Cardiovasc Res. 2011;91:203-11.

16. Hang CT, Yang J, Han P, et al. Chromatin regulation by Brg1 underlies heart muscle development and disease. Nature. 2010;466:62-7.

17. Lickert H, Takeuchi JK, Von Both I, et al. Baf60c is essential for function of BAF chromatin remodelling complexes in heart development. Nature. 2004;432:107-12.

18. Takeuchi JK, Bruneau BG. Directed transdifferentiation of mouse mesoderm to heart tissue by defined factors. Nature. 2009;459:708-11.

19. Takeuchi JK, Lickert H, Bisgrove BW, et al. Baf60c is a nuclear notch signaling component required for the establishment of left-right asymmetry. Proc Natl Acad Sci $U$ S A. $2007 ; 104: 846-51$.

20. Takeuchi JK, Lou X, Alexander JM, et al. Chromatin remodelling complex dosage modulates transcription factor function in heart development. Nat Commun. 2011;2:187-97. 